- PPD2-25 Liquid-liquid equilibria and transport studies of ternary systems composed of ionic liquid, 1-hexanethiol and n-dodecane, A.R. Ferreira, M.G. Freire, L.A. Neves, J.C. Ribeiro, F.M. Lopes, J.G. Crespo and J.A.P. Coutinho (Universidade de Aveiro, Universidade Nova de Lisboa e Galp Energia, Portugal).

Finalmente, é de destacar a excelente participação dos grupos nacionais que investigam os Líquidos lónicos e as suas aplicações no nosso país, desde a Universidade do Porto, passando por Aveiro, Coimbra, e até Lisboa (UL, UTL e UNL).

Por que foi uma workshop no meio académico, contou com a participação ativa de estudantes de $2 .^{\circ}$ e $3 .^{\circ}$ ciclos, o que demonstrou a total aceitação do tema pelos nossos alunos e uma forma de consolidarem matérias dadas em ambiente de sala de aula. Foi possível uma vivência realista, pouco comum no meio académico, e demonstrar que a interdisciplinaridade simbiótica da interação industria-universi- dade é de extrema importância para o desenvolvimento tecnológico atual.

\section{Carlos Nieto de Castro (cacastro@fc.ul.pt) \\ Comissão Organizadora}

\footnotetext{
Grup d'Investigació d'Enginyeria Tèrmica Aplicada, do Departamento de Engenharia Mecânica da Universitat Rovira i Virgili, Tarragona, Espanha.

O objetivo da EUROTHERM é a promoção da Cooperação Europeia em Ciências Térmicas e Transferência de Calor, através da realização de ações de discussão e divulgação em que se envolvam cientistas e engenheiros (http://www.eurothermcommittee.eu). Tanto Carlos Nieto de Castro, como Alberto Coronas, são membros português e espanhol do Eurotherm Committee.
}

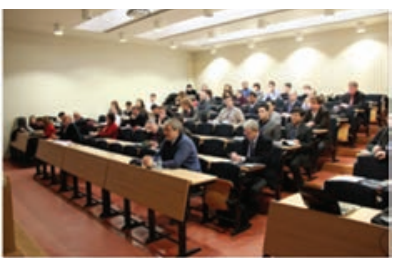

Aspeto da audiência

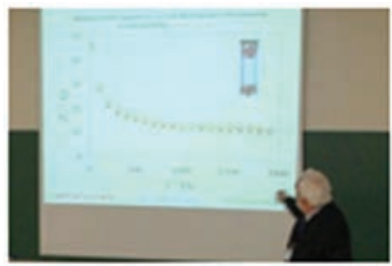

João Fareleira (IST)

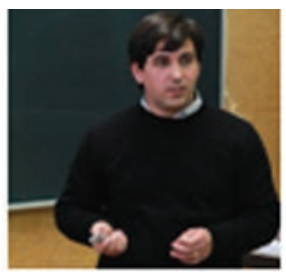

José Esperança (ITQB)

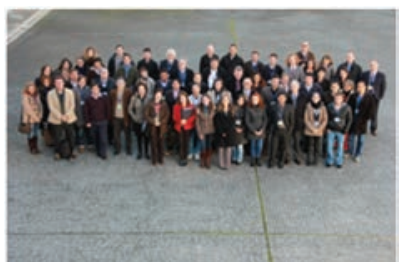

Fotografia de grupo

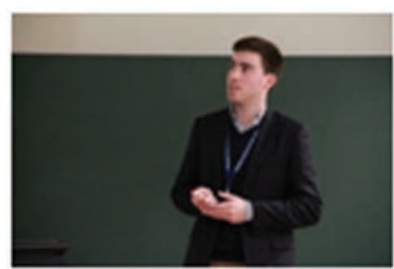

António Ferreira (FCTUC)

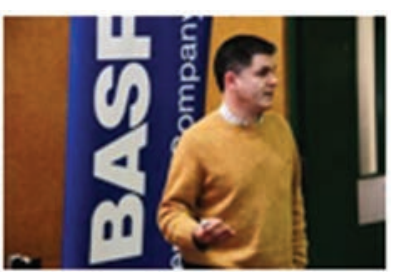

João Coutinho (UA)

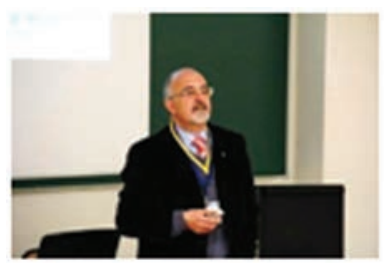

Carlos Nieto de Castro (FCUL)

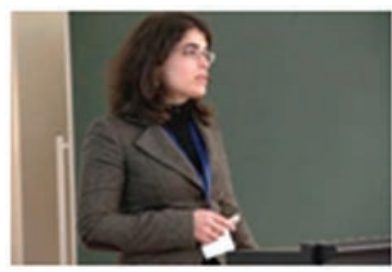

Rita Frade (FFUL)

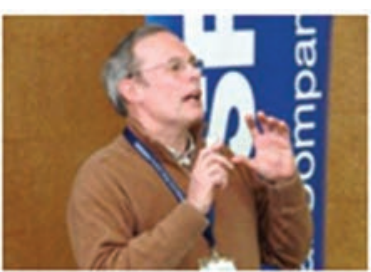

Fernando Santos (FCUL)

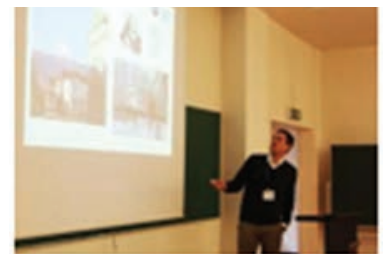

Agílio Pádua (UBP)

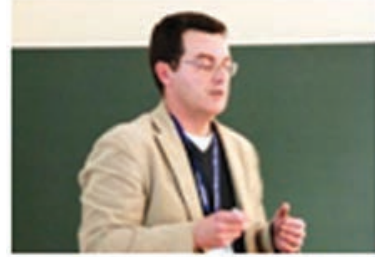

Luís Santos (FCUP)

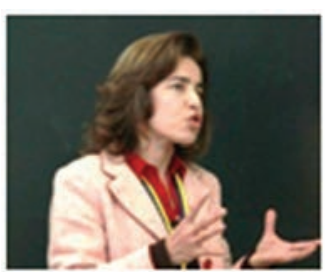

Maria José Lourenço (FCUL)

\title{
International Conference on Solid Compounds of Transition Elements (SCTE2012)
}

Decorreu na Faculdade de Ciências da Universidade de Lisboa, entre 31 de março e 5 de abril de 2012, a conferência " $18^{\text {th }}$ International Conference on Solid Compounds of Transition Elements" (SCTE2012). O evento contou com a participação de mais de 200 participantes (Professores Universitários, Investigadores e Estudantes), originários de cerca de trinta países.
Nesta conferência foram apresentadas 5 lições plenárias, 7 keynotes, 52 comunicações orais e 146 painéis. Os trabalhos versaram essencialmente a química e física dos compostos intermetálicos, óxidos, hidretos, pnictetos e calcogenetos, tendo sido ainda reportados alguns estudos em organometálicos com propriedades magnéticas, óticas, elétricas, biológicas e catalíticas anormais.
O congresso iniciou-se com a lição plenária do Professor Hideo Hosono, do Instituto de Tecnologia de Tóquio, intitulada "Iron pnictide superconductors and relevant functional materials", onde foi feita uma revisão dos recentes progressos na investigação deste tipo de compostos. O Professor Hosono tem-se dedicado ao estudo dos materiais inorgânicos e dos óxidos semicondutores com hiatos elevados, 
tendo recebido vários prémios, como o Achievement Awards da Sociedade de Química do Japão (2004) e o The Bernd T. Matthias Prize (2009), devendo-se a ele a descoberta, em 2008, dos pnictetos supercondutores de Fe. A preparação de pnictetos supercondutores de Fe foi descrita na keynote intitulada "Improved high pressure flux growth, structural, and superconducting properties of $\mathrm{LnFeAsO}$ ( $\mathrm{Ln}=$ $\mathrm{Pr}, \mathrm{Nd}, \mathrm{Sm}$ ) single crystals", proferida pelo Professor Nikolai Zhigadlo, do ETH de Zurique.

Um dos momentos altos deste Congresso foi a Sessão Especial dedicada ao Prémio Nobel da Química 2011. A keynote desta sessão, intitulada "Complex intermetallics - simpler than you think", foi realizada pelo Professor Walter Steurer, do ETH de Zurique, a quem muito se deve no trabalho pioneiro sobre a estrutura atómica, formação e estabilidade dos quasicristais. A lição proferida mostrou de uma maneira simples como construir estruturas quasicristalinas a partir de agregados atómicos e a necessidade de utilizar dimensões superiores a três para descrever as suas estruturas. Em 2008, o Professor Steurer recebeu o prémio Jean Marie Dubois for Excellence in Quasicrystal Research, tendo sido até 2010 o Editor Chefe da revista "Zeitschrift für Kristallographie".

Foram também realizadas as seguintes apresentações plenárias e keynotes:

- "PAC for intermetallic hydrides", Professor João Gil, Universidade de Coimbra;
- "Magnetic properties of selected MM'X-type $\left(\mathrm{M}=\right.$ =metal $3 \mathrm{~d}, \mathrm{M}^{\prime}=$ metal $3 d$ or $4 d X=A s, P, G e, S i)$ intermetallics crystallize in hexagonal or orthorhombic crystal structure", Professor Ryszard Zach, Universidade Politécnica de Cracóvia;

- "Cu, Pu and Fe high TC superconductors: all the same mechanism", Professor Peter Wachter, ETH de Zurique:

- "Exotic Magnetism in Pt and Au nanoparticles", Professor Juan Bartolomé, Universidade de Zaragoza;

- "Hydrogenation and electronic state of cerium", Doutor Bernard Chevalier, Universidade de Bordéus;

- "Group 4 diboride - Ni interactions: a thermodynamic database for the B-Hf-Ni-Ti-Zr system", Professor Gabriele Cacciamani, Universidade de Génova:

- "Relation between microstructure/ nanostructure and stress development in TM-doped Mg-based alloys", Professora Nataliya Skryabina, Universidade Estatal de Perm, Rússia;

onde foi dada especial ênfase à relação entre a estrutura e as propriedades físicas dos compostos.

A complexidade das ligações químicas em intermetálicos, boretos e carbonetos metálicos foi o tema da keynote "Metal-metal bonding in transition metal compounds: a direct space perspective", realizada pelo Doutor Frank Wagner, do Max-Planck-Institut für Chemische Physik fester Stoffe, Dresden. O Professor Mercouri G. Kanatzidis, da Universidade de Northwestern e do Laboratório Nacional de Argonne, fechou a Conferência com a lição plenária "Metallic fluxes as a powerful media for discovery of complex intermetallics“, dedicada à síntese de intermetálicos, onde realçou a grande complexidade da sua química quando comparada com a dos compostos orgânicos.

Os temas de estudo do Professor Kanatzidis têm sido variados, destacando-se a química inorgânica, de estado sólido e de coordenação, e a química exploratória de compostos intermetálicos. Recebeu numerosos prémios, sendo de salientar a Morley Medal, da secção de Cleveland da Sociedade Americana de Química (2003) e a MRS Fellow, Sociedade de Investigação de Materiais (2010). É atualmente o Editor Chefe da revista "Journal of Solid State Chemistry".

A próxima SCTE realizar-se-á na região de Génova, Itália, em maio de 2014.

\section{Agradecimentos}

A SCTE2012 contou com o apoio do European C-MAC (www.eucmac. eu), Termolab (www.termolab.pt), PANalytical (www.panalytical.com), El Corte Inglés (www.elcorteingles. pt), Sumol+Compal (www.sumolcompal.pt) e Divisão de Turismo da Câmara Municipal de Sintra (www. cm-sintra.pt).

A.P. Gonçalves, R.P. Borges, M.D. Carvalho, E.B. Lopes, L.C.J. Pereira e Yu. Verbovytskyy (apg@itn.pt) Comité de Organização

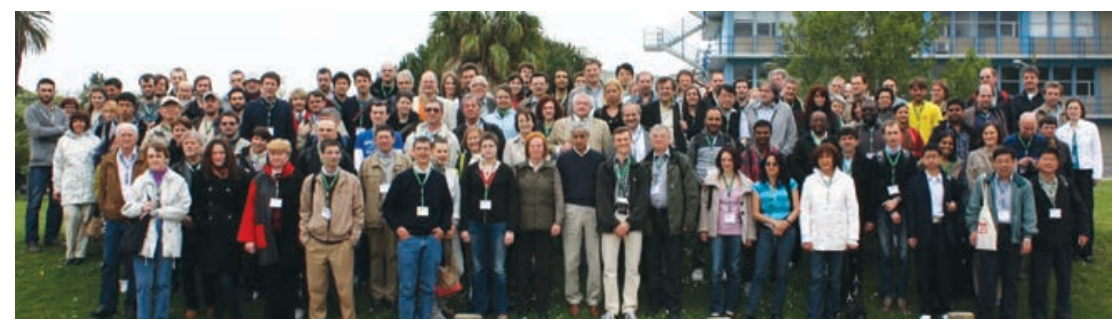

Fotografia de grupo dos participantes no SCTE2012

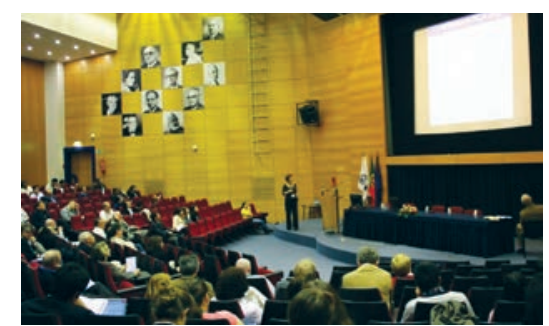

Visão geral do auditório

Visite-nos em www.spq.pt 